\title{
Diagnostic value of myocardial deformation pattern in children with noncompaction cardiomyopathy
}

\begin{abstract}
The current echocardiographic diagnostic criteria for noncompaction cardiomyopathy (NCC) have variable sensitivity and low specificity. Moreover, there are limited data on the use of myocardial deformation imaging for early detection of myocardial dysfunction in children with NCC. We describe left ventricular (LV) deformation patterns in children with NCC, with the goal of identifying a potential diagnostic pattern. We prospectively enrolled 30 children with NCC (47\% male; mean age 7.2 years) and 30 age- and gender-matched controls. Extent and severity of non compaction in each segment were evaluated in LV 16segment model. Regional (base, mid and apex) and segmental (16 segments) longitudinal strain (LS), circumferential strain (CS) and radial strain (RS) were measured using speckle tracking echocardiography. In all patients with NCC, regional and segmental CS and RS at the apex were significantly decreased compared to controls (CS apex: $-19.2 \pm 5.4 \%$ vs. $-30.2 \pm 6.9 \%, \mathrm{p}<0.001 / \mathrm{RS}$ apex: $23.5 \pm 8.6 \%$ vs. $44.1 \pm 14.5 \%, \mathrm{p}<0.001)$. Thirty percent $(9 / 30)$ of patients had an $\mathrm{EF}<50 \%$. In these patients, there was additional decrease in CS in basal segments and in LS in basal, mid-cavity and apical segments (CS base: $-16.4 \pm 4.7 \%$ vs. $-24.6 \pm 3.9 \%, \mathrm{p}<0.001 / \mathrm{LS}$ (average all LV segments): $-13.9 \pm 3.1 \%$ vs. $-20.7 \pm 4.7 \%$, $\mathrm{p}<0.001$ ). A cut-off value of CS at the apex of $-24.5 \%$ was a strong differentiating feature between patients with NCC and EF $>50 \%$ and controls (sensitivity: $87 \%$, specificity $79 \%$, AUC $0.88, \mathrm{p}<0.001)$. Children with NCC exhibit a deformation pattern characterized by decreased apical circumferential strain, which may serve as a potential diagnostic tool for NCC. The role of decreased global LV longitudinal and basal circumferential strain should be further evaluated as a potential prognostic tool.
\end{abstract}

Keyword: Cardiomyopathy; Myocardial deformation; Noncompaction; Speckle tracking echocardiography 